\title{
Radiomic Analysis for Pretreatment Prediction of Recurrence After Radiotherapy in Locally Advanced Cervical Cancer
}

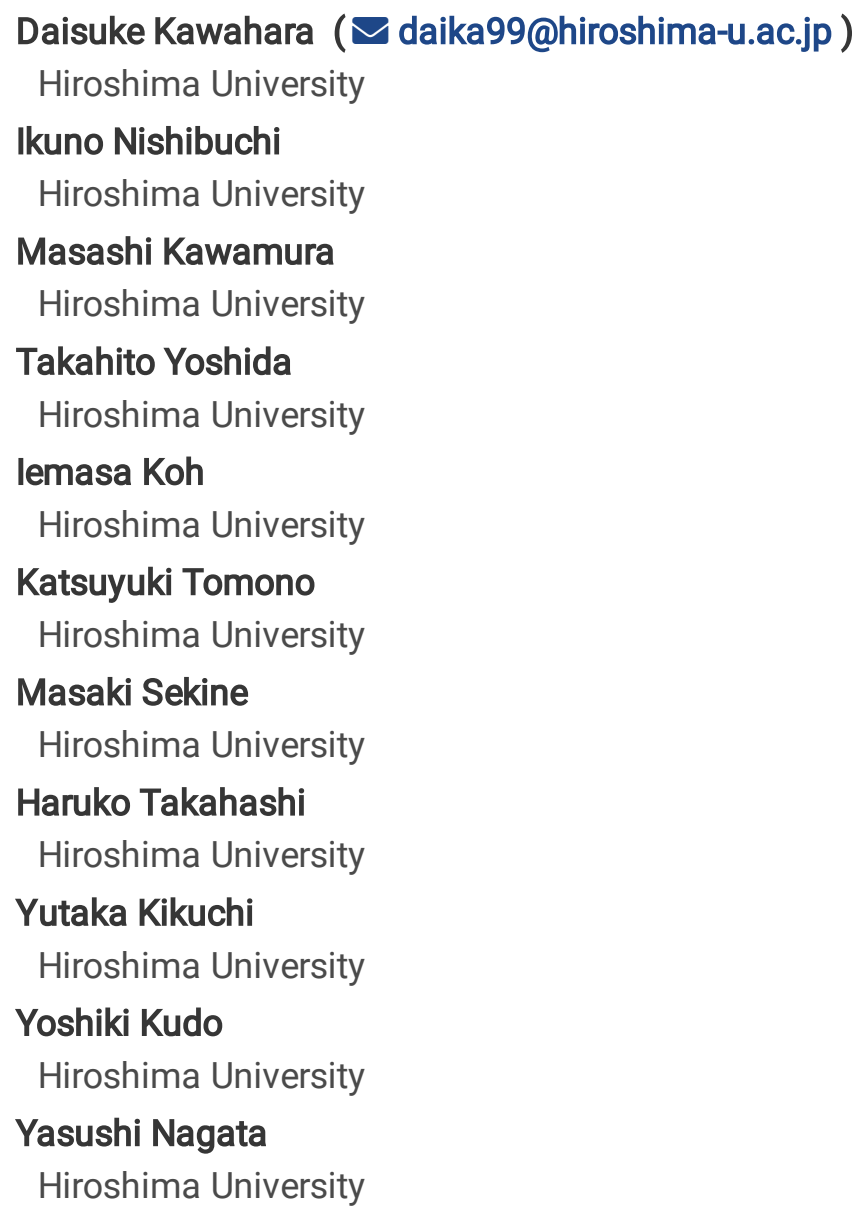

\section{Research Article}

Keywords: Radiotherapy, machine learning, cervix cancer

Posted Date: January 31st, 2022

DOI: https://doi.org/10.21203/rs.3.rs-1198222/v1

License: (c) (i) This work is licensed under a Creative Commons Attribution 4.0 International License. Read Full License

Version of Record: A version of this preprint was published at International Journal of Radiation Oncology*Biology*Physics on November 1st, 2021. See the published version at https://doi.org/10.1016/j.ijrobp.2021.07.477. 


\section{Abstract}

口Objective $\mathbb{X}$ To predict the recurrence of advanced cervical cancer patients treated with radiotherapy from radiomics features on pre-treatment T1- and T2-weighted MRI images.

पMethods $₫$ A total of 90 patients were split into two sets: 67 patients for model training and 23 patients for model testing. The patient outcome was classified into two groups; patients with a recurrence (group I) and without a recurrence (group II). The predictors were selected using the least absolute shrinkage and selection operator (LASSO) regression. The machine learning for the predictive models was sued neural network classifiers. The accuracy, sensitivity, specificity, and the area under the curve (AUC) from the receiver operating characteristic were evaluated.

$\square$ Results $\square$ By the LASSO analysis, we found 25 radiomics features from the T1-weighted MRI image and 4 radiomics features from the T2-weighted MRI image. The accuracy of the prediction model was highest with the combination of T1and T2-weighted MRI images. The model performances with T1-weighted MRI image and T2-weighted MRI image were $86.4 \%$ and $89.4 \%$ of accuracy, $74.9 \%$ and $38.1 \%$ of sensitivity, $81.8 \%$ and $72.2 \%$ of specificity, and 0.89 and 0.69 of AUC. The model performance was improved with the combination of T1- and T2-weighted MRI images, which was $93.1 \%$ of accuracy, $81.6 \%$ of sensitivity, $88.7 \%$ of specificity, and 0.94 of AUC.

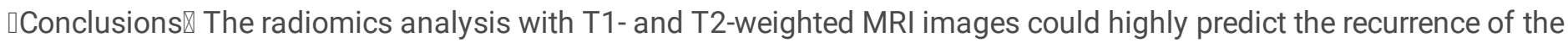
cervix cancer after radiotherapy. The variation of the distribution and the difference of the pixel number at the peripheral and the center were important predictors.

\section{Key Points}

- A MRI-based radiomics model for the prediction of the recurrence of advanced cervical cancer patients treated with radiotherapy was proposed.

- - The accuracy of the prediction model was highest with the combination of T1- and T2-weighted MRI images.

- - The model performance was improved with the combination of T1- and T2-weighted MRI images, which was $93.1 \%$ of accuracy, $81.6 \%$ of sensitivity, $88.7 \%$ of specificity, and 0.94 of AUC.

\section{Introduction}

Cervical cancer is one of the most frequent malignant tumors in worldwide females [1]. Definitive radiotherapy is the mainstream of cervical squamous cell carcinoma for both early-stage and advanced cases. In early-stage, the treatment outcomes of radiotherapy and surgery are comparable. For locally advanced unresectable cervix cancer, concurrent chemoradiotherapy (CCRT) is the standard treatment. However, Jemal et al reported that one-third of patients would experience recurrence [2]. After primary therapy, tumor recurrence is frequently not detected for several months. It is a major challenge to predict the treatment response and the long-term outcome presents for a precise personalized care. In clinical, patients can take the treatment such as additional chemotherapy and dose escalation in time for those patients by predicting reliable biomarkers that can early identify the high-risk recurrence patients.

A medical image such as magnetic resonance imaging (MRI) and computed tomography is essential in the staging of patients and guiding treatment decisions. For cervical cancer patients, the MRI provides high soft-tissue contrast and functional information, which plays a key role in the assessment of the reference standard for the pre-therapeutic [3].

Radiomics analysis provides high-dimensional data such as tumor homogeneity and heterogeneity that cannot be identified by general visual evaluation using texture analysis in addition to the shape and volume [4, 5]. Texture analysis can evaluate the position of the pixels and gray-level intensity within an image using a variety of mathematical methods. 
Radiomics can classify the stage or histology of the tumor through the prediction of responses to chemotherapy [6] or radiotherapy [7].

Reuze et al reported that the PET texture analysis could predict the recurrence for locally advanced cervical cancer treated by brachytherapy and chemoradiation than SUVmax [8]. Meng et al evaluated a useful of texture features extracted from T2-weighted MRI image and apparent diffusion coefficient (ADC) maps for prediction of recurrence for advanced cervical cancer patients treated with concurrent chemoradiotherapy [9]. The prediction model with MRI image improved the accuracy of the prediction than that with PET image. On the other hand, the region of interests (ROIs) were drawn on each slice covering the whole tumor. Xie et al showed usability of a sub-region-based radiomics analysis in which the ROI was divided into sub-regions based on the local entropy and cluster of CT values [10]. It suggests that the number of radiomics features could be increased by adding the ROI and it could improve the accuracy of the prediction.

The current study proposed the prediction model of the recurrence for cervical cancer patients treated e radiomics features using radiomics features extracted from the extended and shrink-uterus regions on pre-treatment T1- and T2-weighted MRI images.

\section{Materials And Methods}

\section{A) Patients}

Eighty-nine cervical squamous cell carcinoma patients who were treated with external beam radiotherapy (EBRT) followed by intracavitary brachytherapy (ICBT) at our institution from 2003 to 2015 were reviewed. All patients provided written informed consent for treatment. The patients and tumor characteristics are presented in Table 1. Among the M1 cases, patients with para-aortic lymph node (PAN) metastasis were included. Hiroshima University Certified Review Board approved this retrospective study (E-1656). The need for informed consent was waived owing to the retrospective nature of the study. The methods in the current study were performed according to relevant regulations and guidelines. 
Table 1

Patient and tumor characteristics

\begin{tabular}{|lll|}
\hline Age (years) & Median (range) & $\mathbf{6 3}$ (30-85) \\
\hline PS & 0 & 71 \\
& 1 & 14 \\
\hline & 2 & 4 \\
\hline Histology & 3 & 0 \\
\hline T factor (UICC-8th) & Squamous & 89 \\
& $1 \mathrm{a}$ & 1 \\
\hline & $1 \mathrm{~b}$ & 8 \\
\hline $2 \mathrm{a}$ & 1 \\
\hline 2b & 37 \\
\hline 3a & 0 \\
\hline N factor & $3 \mathrm{~b}$ & 36 \\
\hline M factor & $4 a$ & 6 \\
\hline & 0 & 43 \\
\hline 1 & 46 \\
\hline & 0 & 76 \\
\hline
\end{tabular}

\section{B) Image Acquisition}

MRI images were scanned with three 1.5 T MRI imaging units (Integenia Ambition, Philips; Siemens Healthcare Magnetom Avanto; Signa Excite, GE Healthcare), with a pelvic array coil for the pelvic scans. All patients were scanned using the same MR sequence, including axial T1-weighted fast spin-echo (FSE), and axial T2-weighted FSE. The patients who scanned only T1- or T2-weighted FSE with fat saturation were eliminated in the analysis. Images from 89 patients were retrospectively analyzed under an institutional-review-board-approved study.

\section{C) Treatment Radiotherapy}

One patient was treated with ICBT alone, and 88 patients were treated with a combination of EBRT and ICBT. Threedimensional radiotherapy planning was performed for all the patients who received EBRT using an X-ray beam (6-18 MV). Patients without PAN metastasis received whole pelvis irradiation (WPI) and patients with PAN metastasis received extended-field irradiation. Center shielding (CS) was used in 67 patients and boost irradiation for lymph node or parametrium regions was performed in 36 patients. The indication and dose of CS and boost irradiation were ultimately determined by the radiation oncologist based on the initial tumor size and therapeutic effect of WPI. The median EBRT dose was $50 \mathrm{~Gy} / 25$ fractions (range 28-66 Gy). Image-guided brachytherapy (IGBT) was performed in 20 patients. The prescribed dose of ICBT was $6 \mathrm{~Gy}$ to point A (a point $2 \mathrm{~cm}$ cranial from the external cervical os and $2 \mathrm{~cm}$ lateral from the 
tandem) in two-dimensional treatment planning and to D90 (minimum dose to the $90 \%$ ) of high-risk clinical target volume (the residual tumor at the time of ICBT and the whole uterine cervix) in IGBT. The fractions of ICBT depended on CS dose and the median ICBT dose was $18 \mathrm{~Gy} / 3$ fractions (range 6-30 Gy/1-5 fractions). The median overall treatment time was 44 days (range $30-58$ days). The most common treatment schedule was as follows: whole pelvis irradiation $40 \mathrm{~Gy} / 20$ fractions, CS 10 Gy/5 fractions, and ICBT 18 Gy/3 fractions.

\section{Chemotherapy}

Sixty-eight patients received concurrent chemotherapy. According to our hospital's protocol and the physician's judgment, the selection of chemotherapeutic regimen and reduction of chemotherapeutic dosages were determined. The regimens of the chemotherapeutic were as follows: weekly cisplatin in 33 patients, weekly nedaplatin in 21 patients, intraarterial chemotherapy in 10 patients, and others in four patients.

\section{F) Radiomics Analysis}

The process acquisition of the MRI images to prediction model is shown in Fig. 1. The proposed radiomics model was designed as a Transparent Reporting of a Prediction Model for Individual Prognosis or Diagnosis (TRIPOD) type 2a [11]. The T1- and T2-weighted The MRI images were transferred to a medical image computing tool (3D Slicer, www.slicer.org) [12]. Fig. 2 shows the example of the segmentation. Uterus included the primary tumor was defined as CTV was manually segmented on the axial T1- and T2-weighted MRI images. The segmentation was performed by one or two radiation oncologists, including one expert radiation oncologist Then, the extended-CTVs were generated by adding $5 \mathrm{~mm}, 10 \mathrm{~mm}$, and $20 \mathrm{~mm}$ margins from the CTV, which were defined as eCTV5, eCTV10, and eCTV20. Moreover, shrink-CTVs were generated by adding $5 \mathrm{~mm}$ and $10 \mathrm{~mm}$ margins from the CTV, which were defined as SCTV5 and sCTV10. The radiomics features were extracted with an open-source package in Python, Pyradiomics software [13]. A detailed list of the radiomics features is shown in Table 2 and Table 3. 
Table 2

Feature type and associated features

\begin{tabular}{|c|c|c|c|c|c|c|c|}
\hline $\begin{array}{l}\text { Feature } \\
\text { type }\end{array}$ & $\begin{array}{l}\text { Morphology } \\
\text {-based }\end{array}$ & $\begin{array}{l}\text { First order- } \\
\text { based }\end{array}$ & Texture-based & & & & \\
\hline Methods & Shape & Histogram & GLCM & GLSZM & GLRLM & NGTDM & GLDM \\
\hline \multirow[t]{12}{*}{$\begin{array}{l}\text { Feature } \\
\text { name }\end{array}$} & $\begin{array}{l}\text { Maximum } \\
\text { 3D } \\
\text { diameter }\end{array}$ & $\begin{array}{l}\text { Interquartile } \\
\text { range }\end{array}$ & Joint average & $\begin{array}{l}\text { Gray level } \\
\text { variance }\end{array}$ & $\begin{array}{l}\text { Short run } \\
\text { low gray } \\
\text { level } \\
\text { emphasis }\end{array}$ & Coarseness & $\begin{array}{l}\text { Gray level } \\
\text { variance }\end{array}$ \\
\hline & $\begin{array}{l}\text { Maximum } \\
2 \mathrm{D} \\
\text { diameter } \\
\text { slice }\end{array}$ & Skewness & Sum average & $\begin{array}{l}\text { Zone } \\
\text { variance }\end{array}$ & $\begin{array}{l}\text { Gray level } \\
\text { variance }\end{array}$ & Complexity & $\begin{array}{l}\text { High gray } \\
\text { level } \\
\text { emphasis }\end{array}$ \\
\hline & Sphericity & Uniformity & Joint entropy & $\begin{array}{l}\text { Gray level } \\
\text { non } \\
\text { uniformity } \\
\text { normalized }\end{array}$ & $\begin{array}{l}\text { Low gray } \\
\text { level run } \\
\text { emphasis }\end{array}$ & Strength & $\begin{array}{l}\text { Dependence } \\
\text { entropy }\end{array}$ \\
\hline & Minor axis & Median & Cluster shade & $\begin{array}{l}\text { Size zone } \\
\text { non } \\
\text { uniformity } \\
\text { normalized }\end{array}$ & $\begin{array}{l}\text { Gray level } \\
\text { non } \\
\text { uniformity } \\
\text { normalized }\end{array}$ & Contrast & $\begin{array}{l}\text { Dependence } \\
\text { non } \\
\text { uniformity }\end{array}$ \\
\hline & Elongation & Energy & $\begin{array}{l}\text { Maximum } \\
\text { probability }\end{array}$ & $\begin{array}{l}\text { Size zone } \\
\text { non } \\
\text { uniformity }\end{array}$ & $\begin{array}{l}\text { Run } \\
\text { variance }\end{array}$ & Busyness & $\begin{array}{l}\text { Gray level } \\
\text { non } \\
\text { uniformity }\end{array}$ \\
\hline & $\begin{array}{l}\text { Surface } \\
\text { volume } \\
\text { ratio }\end{array}$ & $\begin{array}{l}\text { Robust } \\
\text { mean } \\
\text { absolute } \\
\text { deviation }\end{array}$ & Idmn & $\begin{array}{l}\text { Gray level } \\
\text { non } \\
\text { uniformity }\end{array}$ & $\begin{array}{l}\text { Gray level } \\
\text { non } \\
\text { uniformity }\end{array}$ & & $\begin{array}{l}\text { Small } \\
\text { dependence } \\
\text { emphasis }\end{array}$ \\
\hline & Volume & $\begin{array}{l}\text { Mean } \\
\text { absolute } \\
\text { deviation }\end{array}$ & Joint energy & $\begin{array}{l}\text { Large area } \\
\text { emphasis }\end{array}$ & $\begin{array}{l}\text { Long run } \\
\text { emphasis }\end{array}$ & & $\begin{array}{l}\text { Small } \\
\text { dependence } \\
\text { high gray } \\
\text { level } \\
\text { emphasis }\end{array}$ \\
\hline & Major axis & $\begin{array}{l}\text { Total } \\
\text { energy }\end{array}$ & Contrast & $\begin{array}{l}\text { Small Area } \\
\text { high gray } \\
\text { level } \\
\text { emphasis }\end{array}$ & $\begin{array}{l}\text { Short Run } \\
\text { high gray } \\
\text { level } \\
\text { emphasis }\end{array}$ & & $\begin{array}{l}\text { Dependence } \\
\text { non } \\
\text { uniformity } \\
\text { normalized }\end{array}$ \\
\hline & $\begin{array}{l}\text { Surface } \\
\text { area }\end{array}$ & Maximum & $\begin{array}{l}\text { Difference } \\
\text { entropy }\end{array}$ & $\begin{array}{l}\text { Zone } \\
\text { percentage }\end{array}$ & $\begin{array}{l}\text { Run length } \\
\text { non } \\
\text { uniformity }\end{array}$ & & $\begin{array}{l}\text { Large } \\
\text { dependence } \\
\text { emphasis }\end{array}$ \\
\hline & Flatness & $\begin{array}{l}\text { Root mean } \\
\text { squared }\end{array}$ & $\begin{array}{l}\text { Inverse } \\
\text { variance }\end{array}$ & $\begin{array}{l}\text { Large area } \\
\text { low gray } \\
\text { level } \\
\text { emphasis }\end{array}$ & $\begin{array}{l}\text { Short run } \\
\text { emphasis }\end{array}$ & & $\begin{array}{l}\text { Large } \\
\text { dependence } \\
\text { low gray } \\
\text { level } \\
\text { emphasis }\end{array}$ \\
\hline & Least axis & $\begin{array}{l}90 \\
\text { percentile }\end{array}$ & $\begin{array}{l}\text { Difference } \\
\text { variance }\end{array}$ & $\begin{array}{l}\text { Large area } \\
\text { high gray } \\
\text { level } \\
\text { emphasis }\end{array}$ & $\begin{array}{l}\text { Long run } \\
\text { high gray } \\
\text { level } \\
\text { emphasis }\end{array}$ & & $\begin{array}{l}\text { Dependence } \\
\text { variance }\end{array}$ \\
\hline & $\begin{array}{l}\text { Maximum } \\
2 \mathrm{D} \\
\text { diameter } \\
\text { column }\end{array}$ & Minimum & Idn & $\begin{array}{l}\text { High gray } \\
\text { level zone } \\
\text { emphasis }\end{array}$ & $\begin{array}{l}\text { Run } \\
\text { percentage }\end{array}$ & & $\begin{array}{l}\text { Large } \\
\text { dependence } \\
\text { high gray } \\
\text { level } \\
\text { emphasis }\end{array}$ \\
\hline
\end{tabular}




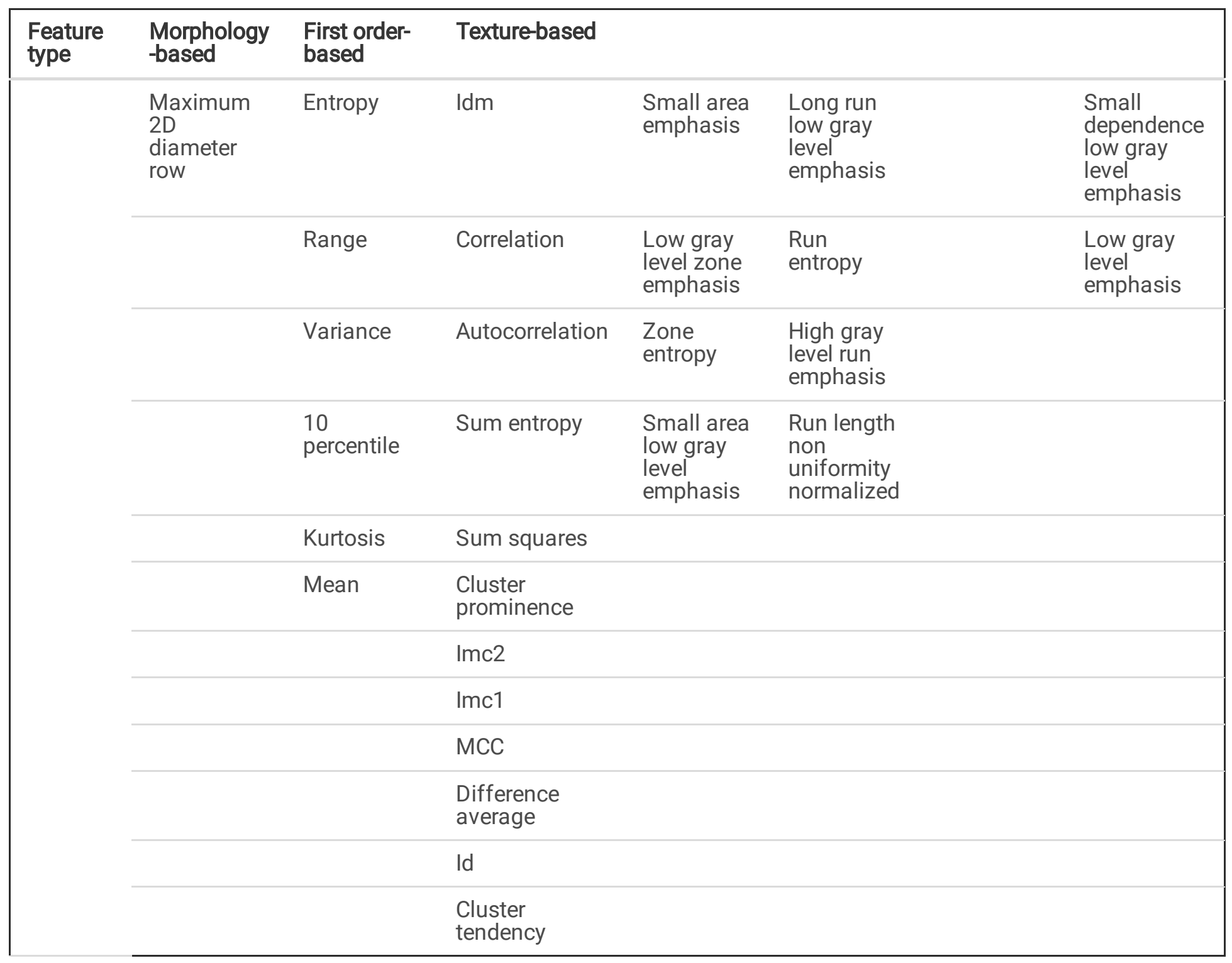

Table 3

Feature associated with the imaging filters

\begin{tabular}{|ll|}
\hline $\begin{array}{l}\text { Feature } \\
\text { type }\end{array}$ & Wavelet-based \\
\hline Methods & $\begin{array}{l}\text { First-order statistic and texture of wavelet decomposition. Decomposition levels: LLL, LLH, LHL, LHH, } \\
\text { HLL, HLH, HHL, HHH. }\end{array}$ \\
\hline $\begin{array}{l}\text { Feature } \\
\text { name }\end{array}$ & $\begin{array}{l}\text { First-order features } \\
\text { GLCM features }\end{array}$ \\
\hline & GLSZM features \\
& GLRLM features \\
\cline { 2 - 2 } & NGTDM features \\
\hline & GLDM features \\
\hline
\end{tabular}

We extracted 13 shape radiomics features, 21 first-order radiomics features, 50 quantitative radiomics features, and 93 texture radiomics features. Additionally, the radiomics features were extracted from the wavelet filters which has high-pass 
and low-pass filters. The wavelet filter was decomposed in $x, y$, and $z$ direction. A total of 837 radiomics features were extracted for each segmentation.

\section{G) Prediction Model}

In this study, all loco-regional recurrence and distant metastasis after RT were regarded as recurrence and examined the prediction model for recurrence. The clinical patient data were updated in May 2020 and the median follow-up time was 59 months (range, 1-160 months). At the time of the last follow-up, recurrence was observed in 30 of 89 patients.

The least absolute shrinkage and selection operator (LASSO) regression model was used with MATLAB code to prevent overfitting $[14,15]$. The most significant predictive features were selected with the LASSO regression which reduces the dimension from among all the candidate features in the training dataset.

We classified recurrence patients and non-recurrence patients with $\mathrm{ML}$ classifiers. The recurrence and non-recurrence patients were labeled as 1 and 0 , respectively. The machine learning (ML) classifiers used a neural network (NN) with rectified linear unit activation and 10 hidden layers. All patients were randomly divided into a training set (49 patients), a validation set (14 patients), and a testing set (26 patients). The prediction model was constructed with the five-fold crossvalidation method, as shown in Fig. 3. The predictive performance was evaluated using the area under the curve (AUC) from the receiver operator characteristic (ROC) curve, accuracy, sensitivity, and specificity.

\section{Results}

A total of 5022 features were extracted from T1-weighted MRI images and the T2-weighted MRI images, respectively. T1weighted MRI image was finally reduced to 25 features and the T2-weighted MRI image was finally reduced to 4 features with the LASSO regression model, as shown in Fig. 4 and Table 4. The following features were extracted from the T1weighted MRI image: 1 feature from the CTV, 2 features from the eCTV5, 7 features from the eCTV20, 5 features from the sCTV5, 10 features from the sCTV10. From the T2-weighted MRI image, 1 feature from the CTV, 1 feature from the eCTV20, 1 feature from the SCTV20, and 1 feature from the SCTV10 were extracted. Most of the features with the wavelet filter were extracted. The T1-weighted MRI image had a large number of features used for the prediction model than the T2-weighted MRI image. The features of the low-pixel number at the center region of the uterus for the T1-weighted MRI image mean the low blood flow. From the shrink-CTV (SCTV) analysis, the radiomics feature of a lower pixel number and a high conformality was selected from the T1- and T2-weighted MRI images. The area of the low pixel number was larger and the conformality was larger with the T1-weighted MRI image for the recurrence group. In the while, the mean value of low pixel number was smaller and the low pixel number with T2-weighted MRI image for the recurrence group. From the extended-CTV, the features that showed a nonuniformity, asymmetric distribution of the pixel values, and a larger volume with the high pixel number were selected. 
Table 4

Selected the features by LASSO Cox regression.

\begin{tabular}{|c|c|c|c|}
\hline ROI & Filter & \multicolumn{2}{|l|}{ Feature list } \\
\hline \multicolumn{4}{|c|}{ T1-weighted MRI image } \\
\hline CTV & wavelet-LLH & Firstorder & Skewness \\
\hline eCTV5 & wavelet-HLL & GLDM & LargeDependenceHighGrayLevelEmphasis \\
\hline eCTV5 & wavelet-HLH & GLCM & Correlation \\
\hline eCTV20 & original & Shape & SurfaceVolumeRatio \\
\hline eCTV20 & original & GLCM & MCC \\
\hline eCTV20 & wavelet-LLH & GLSZM & GrayLevelNonUniformity \\
\hline eCTV20 & wavelet-HLH & Firstorder & Kurtosis \\
\hline eCTV20 & wavelet-HHH & Firstorder & Skewness \\
\hline eCTV20 & wavelet-HHL & Gldm & DependenceNonUniformity \\
\hline eCTV20 & wavelet-HHL & Glcm & Imc1 \\
\hline sCTV5 & wavelet-HLL & Glcm & InverseVariance \\
\hline sCTV5 & wavelet-HLL & Firstorder & Skewness \\
\hline sCTV5 & wavelet-LHL & Glszm & LargeAreaLowGrayLevelEmphasis \\
\hline sCTV5 & wavelet-LLH & Firstorder & Skewness \\
\hline sCTV5 & wavelet-HLH & Firstorder & Median \\
\hline sCTV10 & original & Glszm & LargeAreaHighGrayLevelEmphasis \\
\hline sCTV10 & wavelet-HLL & Glcm & InverseVariance \\
\hline sCTV10 & wavelet-LHH & Firstorder & Mean \\
\hline sCTV10 & wavelet-LLH & Gldm & SmallDependenceLowGrayLevelEmphasis \\
\hline sCTV10 & wavelet-HLH & Firstorder & Mean \\
\hline sCTV10 & wavelet-HHH & Gldm & SmallDependenceLowGrayLevelEmphasis \\
\hline sCTV10 & wavelet-HHH & Firstorder & Skewness \\
\hline sCTV10 & wavelet-HHL & Firstorder & Skewness \\
\hline sCTV10 & wavelet-HHL & Firstorder & Mean \\
\hline sCTV10 & wavelet-LLL & Gldm & SmallDependenceLowGrayLevelEmphasis \\
\hline \multicolumn{4}{|c|}{ T2-weighted MRI image } \\
\hline CTV & wavelet-HHH & Firstorder & Median \\
\hline eCTV20 & wavelet-HLL & Firstorder & Skewness \\
\hline sCTV5 & wavelet-HHH & Firstorder & Median \\
\hline sCTV10 & original & Gldm & SmallDependenceLowGrayLevelEmphasis \\
\hline
\end{tabular}


The prediction models with T1-weighted MRI images, T2-weighted MRI images, and the combination of T1and T2weighted MRI images were evaluated. Figures 5-7 show the validation of the performance of the predictive models according to ROC metrics with 5-fold cross-validation. Table 5 shows the results of the accuracy, sensitivity, specificity, and AUC for the training and testing data.

Table 5

Assessment of the predictive performance of the predictive model for training and testing data with T1-weighted MRI image (T1), T2-weighted MRI image (T2), and the combination of T1- and T2-weighted MRI images (T1\&T2).

\begin{tabular}{|lllllll|}
\hline & T1 & & T2 & & T1\&T2 & \\
\hline & Training & Test & Training & Test & Training & Test \\
\hline Sensitivity & 89.9 & 86.4 & 87.8 & 87.4 & 97.6 & 93.1 \\
\hline Specificity & 81.7 & 74.9 & 31.3 & 38.1 & 92.2 & 81.6 \\
\hline Accuracy & 87.2 & 81.8 & 67.9 & 72.2 & 95.9 & 88.7 \\
\hline AUC & & 0.89 & & 0.69 & & 0.94 \\
\hline
\end{tabular}

The average accuracy of the 5 models for the testing data was $81.8 \%$ with T1-weighted MRI image, $72.2 \%$ with T2weighted MRI image, and $88.7 \%$ with a combination of T1- and T2-weighted MRI images. The average of the 5 models for the testing data was 0.89 with T1-weighted MRI image, 0.69 with T2-weighted MRI image, and $0.94 \%$ with a combination of T1- and T2-weighted MRI images. The prediction model with a combination of T1- and T2-weighted MRI images had higher accuracy and AUC. The prediction model with a T1-weighted MRI image had higher accuracy and AUC than the T2weighted MRI image. Especially, the specificity of the prediction model with T2-weighted MRI images was under $40 \%$ for both training and testing data.

\section{Discussion}

Radiomics approach uses the image-based features as the imaging biomarker for the prediction of the grade of the tumor, treatment response, and side effects by treatment. The past studies (Ho et al, and Reuze et al) reported that the PET texture analysis could predict the recurrence of the cervix cancer than SUVmax $[16,17]$. The AUC of the prediction model with PET texture analysis was 0.75 by Ho et al and 0.76 by Reuze et al Mengal et al improved the accuracy of the prediction of recurrence of advanced cervical cancer patients treated with concurrent chemoradiotherapy using the texture features extracted from T2-weighted MRI image and apparent diffusion coefficient (ADC) maps [18]. The AUC of the prediction model with the support vector machine was 0.89 . Our study demonstrates the potential of radiomics analysis using T1- and T2-weighted MRI images to predict recurrence of cervix cancer after radiotherapy. The current study improved the accuracy by the prediction model of T1- and T2-weighted MRI images with the neural network. The PET is less clinically used than the MRI image and the MRI image had a more predictor.

Sun et al showed a potential of prediction of the clinical response to neoadjuvant chemotherapy with the radiomics analysis of combining the intratumoral and peritumoral regions on the pretreatment T1-and T2-weighted MRI images [19]. The current study investigated the usability of the radiomics model based on pretreatment T1- and T2-weighted MRI images for the prediction of recurrence after radiotherapy. The features of the segmentation of the shrink-CTV and extended-CTV in addition to the CTV were selected for the prediction model. The extended-CTV could extract the features 
in and the boundaries of the tumor, which allows us to detect its associations with metastases within the microenvironment [20].

Nina et al investigated the correlation of the dynamic T1-weighted MRI image for the prediction of tumor control in patients treated with radiotherapy for advanced cervical cancer by pixel by pixel statistical analysis [21]. The dynamic MRI contrast enhancement can assess the regional variation in tumor microcirculation and allow for better assessment of low perfusion regions within tumors. They revealed the correlation of the poor blood supply and hypoxia contribute to radiation therapy failure.

The current study performed the radiomics analysis with T1- and T2-weighted MRI images, not the dynamic MRI contrast enhancement image. The shrink-CTV was mostly limited to the primary tumor region. In the shrink-CTV, the distribution of small dependence with lower pixel values was larger with T1-weighted MRI image and smaller with T2-weighted MRI image. It can suggest that the central tumor region is low blood flow. Thus, the radiomics feature can detect the hypoxia region by poor blood supply without dynamic contras enhanced MRI image. Although in the future, it is needed to validate the correlation of these features and blood flow and hypoxia, we think that the results of this study focused on T1weighted MRI images are of great significance.

There were several limitations in the current study. The current study was conducted at a single institution with a limited number of cervix cancer patients. We consider it necessary to examine universal prediction model with a large number of cases in a multicenter. The current study used multiple MRI devices. A further study will be performed to reveal the robustness of the radiomics features between these devices. The prediction model was proposed with only pretreatment MRI images. Meng et al improved the prediction of the recurrence for cervix cancer using MRI images during treatment [22]. The changes in radiomics features from pretreatment and during treatment, called delta-radiomics features, have been investigated for their prognostic potential in cancer [23-25].

In the future, we reveal the correlation of the radiomics feature and the biological effect and construct a high versatile predictive model.

\section{Conclusion}

The radiomics analysis with T1- and T2-weighted MRI images could highly predict the recurrence of the cervix cancer after radiotherapy. The variation of the distribution and the difference of the pixel number at the peripheral and the center were important predictors.

\section{Declarations}

\section{Acknowledgements}

We gratefully acknowledge The Mathworks, Inc. for providing technical support.

\section{CONFLICT OF INTEREST}

The authors state that there are no conflicts of interest.

\section{AUTHOR CONTRIBUTIONS}

DK and IN are equally-contributed first author. DK and IN conceived and designed the study, and write the manuscript. DK, IN, MK, and YT performed data collection, data analysis and interpretation of results. IK, KT, MS, HT, YK and YN helped interpret the data and write the manuscript. 


\section{References}

1. Siegel R.L. Miller K.D. Jemal A. Cancer statistics, 2019. CA Cancer J Clin. 2019; 69: 7-34.

2. Jemal, A. et al. Global cancer statistics. CA Cancer J Clin. 2011; 61: 69-90.

3. Sala E, Wakely S, Senior E, Lomas D. MRI of malignant neoplasms of the uterine corpus and cervix. AJR Am J Roentgenol. 2007; 188: 1577-87.

4. Zhenyu Liu, Yinyan Wang , Xing Liu, et al. Radiomics analysis allows for precise prediction of epilepsy in patients with low-grade gliomas. Neuroimage Clin, 2018; 19: 271-278.

5. J. Guo, Z. Liu, C. Shen, et al. MR-based radiomics signature in differentiating ocular adnexal lymphoma from idiopathic orbital inflammation Eur Radiol, 2018; 28: 3872-3881.

6. C. Shen, Z. Liu, Z. Wang, J, et al. Building CT radiomics based nomogram for preoperative esophageal cancer patients lymph node metastasis prediction Transl Oncol, 2018: 11: 815-824.

7. Lovinfosse P, Janvary ZL, Coucke P, Jet al. FDG PET/CT texture analysis for predicting the outcome of lung cancer treated by stereotactic body radiation therapy. Eur J Nucl Med Mol Imaging. 2016; 43: 1453-60.

8. Reuzé S, Orlhac F, Chargari $\mathrm{C}$, et al. Prediction of cervical cancer recurrence using textural features extracted from 18FFDG PET images acquired with different scanners. Oncotarget. 2017; 8(26): 43169-43179.

9. Meng J, Liu S, Zhu L, Zhu L, Wang H, Xie L, et al. Texture analysis as imaging biomarker for recurrence in advanced cervical cancer treated with CCRT. Sci Rep. 2018; 8: 11399.

10. Xie C, Yang P, Zhang X, et al. Sub-region based radiomics analysis for survival prediction in oesophageal tumours treated by definitive concurrent chemoradiotherapy. EBioMedicine. 2019; 44: 289-297.

11. Collins GS, Reitsma JB, Altman DG, et al. Transparent reporting of a multivariable prediction model for indi-vidual prognosis or diagnosis (TRIPOD): The TRIPOD statement. Ann. Intern. Med. 2015; 162: 55-63.

12. Fedorov A, Beichel R, Kalpathy-Cramer J, et al. 3D slicer as an image computing platform for the quantitative imaging network. Magn Reson Imaging. 2012;30:1323-1341.

13. Jjm VG, Fedorov A, Parmar C, et al. Computational Radiomics system to decode the radiographic phenotype. Cancer Res. 2017; 77: e104.

14. T ibshirani R. Regression shrinkage and selection via the lasso: a retrospective. J R Stat Soc Series B Stat Methodol. 2011;73:273-82.

15. J.X. Zhang, W. Song, Z.H. Chen, et al. Prognostic and predictive value of a microRNA signature in stage II colon cancer: a microRNA expression analysis. Lancet Oncol. 2013; 14(13): 1295-306.

16. Ho, K. C. et al. A preliminary investigation into textural features of intratumoral metabolic heterogeneity in (18)F-FDG PET for overall survival prognosis in patients with bulky cervical cancer treated with definitive concurrent chemoradiotherapy. Am J Nucl Med Mol Imaging 2016; 6(3): 166-175.

17. Reuzé S, Orlhac F, Chargari $C$, et al. Prediction of cervical cancer recurrence using textural features extracted from $18 \mathrm{~F}-$ FDG PET images acquired with different scanners. Oncotarget. 2017; 8(26): 43169-43179.

18. Meng J, Liu SL, Zhu LJ, et al. Texture Analysis as Imaging Biomarker for recurrence in advanced cervical cancer treated with CCRT. Sci Rep. 2018; 8(1): 11399.

19. Sun C, Tian X, Liu Z, et al. Radiomic analysis for pretreatment prediction of response to neoadjuvant chemotherapy in locally advanced cervical cancer: a multicentre study. EBioMedicine. 2019; 46: 160-169.

20. Pei N, Guangjie Y, Jian G, et al. A CT-based radiomics nomogram for differentiation of focal nodular hyperplasia from hepatocellular carcinoma in the non-cirrhotic liver. Cancer Imaging. 2020; 20: 20.

21. Nina A. Mayr, William T.C. Yuh, Jeffrey C, et al. Pixel analysis of MR perfusion imaging in predicting radiation therapy outcome in cervical cancer. J Magn Reson Imaging. 2000; 12(6): 1027-33.

Page $12 / 16$ 
22. Meng J, Liu S, Zhu L, Zhu L, Wang H, Xie L, et al. Texture analysis as imaging biomarker for recurrence in advanced cervical cancer treated with CCRT. Sci Rep. 2018; 8: 11399.

23. Rao, S. X. et al. CT texture analysis in colorectal liver metastases: A better way than size and volume measurements to assess response to chemotherapy? United Eur. Gastroenterol. J. 2016; 4: 257-263.

24. Goh, V. et al. Assessment of response to tyrosine kinase inhibitors in metastatic renal cell cancer: CT texture as a predictive biomarker. Radiology. 2011; 261: 165-171.

25. Cunliffe, A. et al. Lung texture in serial thoracic computed tomography scans: Correlation of radiomics-based features with radiation therapy dose and radiation pneumonitis development. Int. J. Radiat. Oncol. Biol. Phys. 2015; 91: 10481056.

\section{Figures}

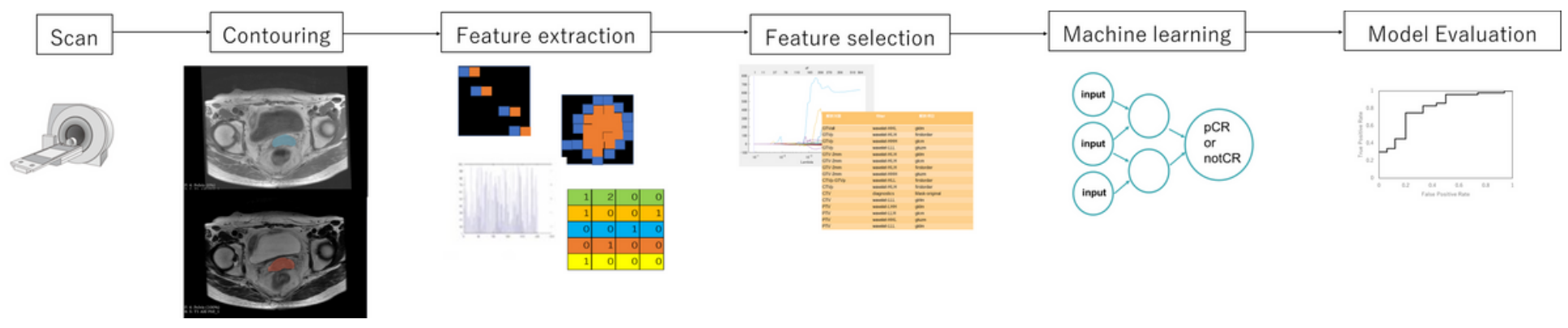

Figure 1

The process of the prediction model with radiomics analysis and machine learning.
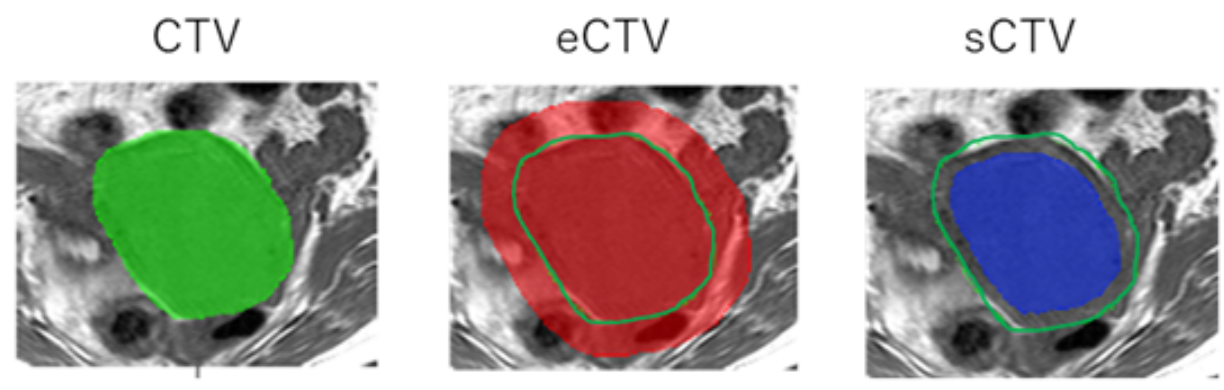

Figure 2

An example of the segmentation of CTV, extended-CTV (eCTV), and shrink-CTV (sCTV). 


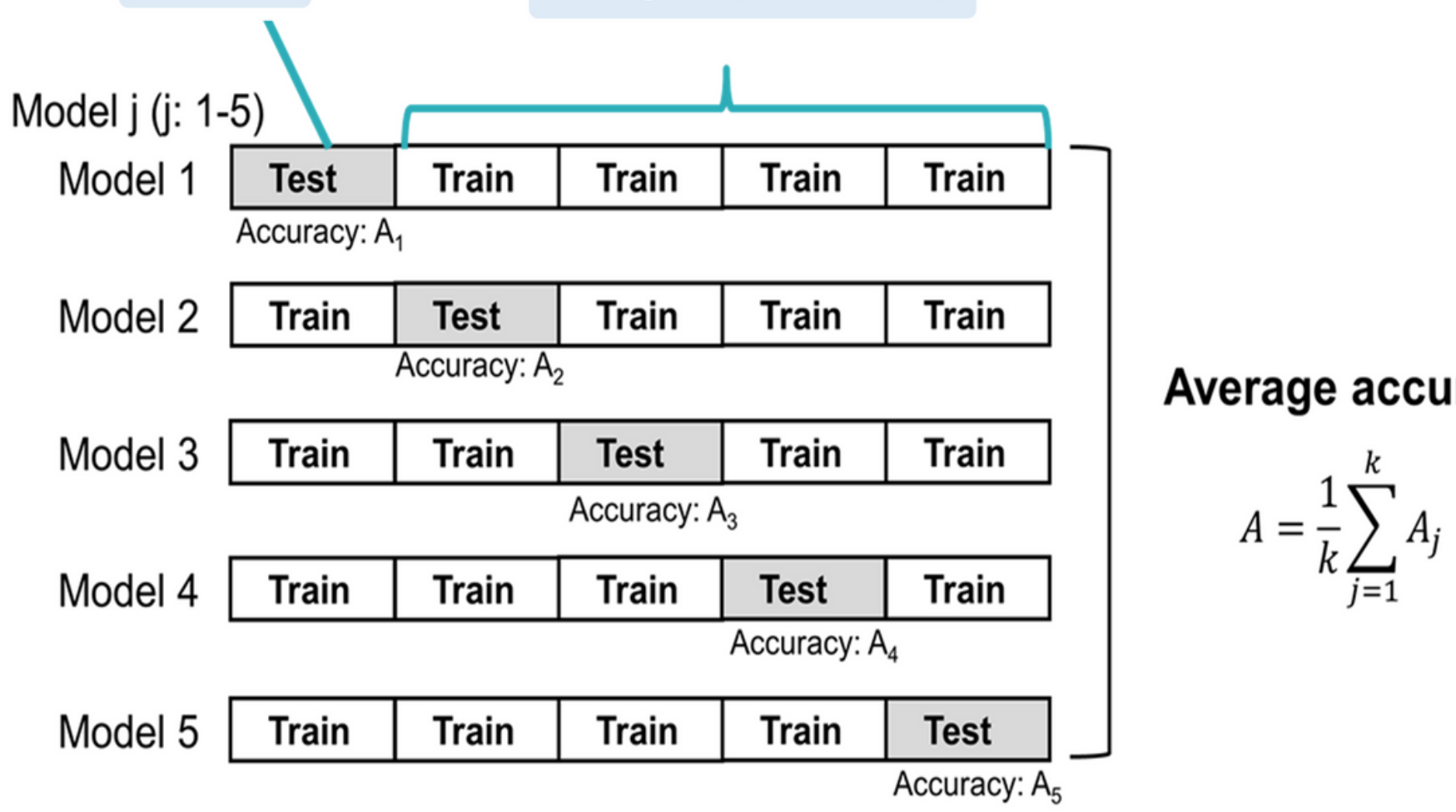

Figure 3

Training and testing with 5-fold cross-validation.

a)

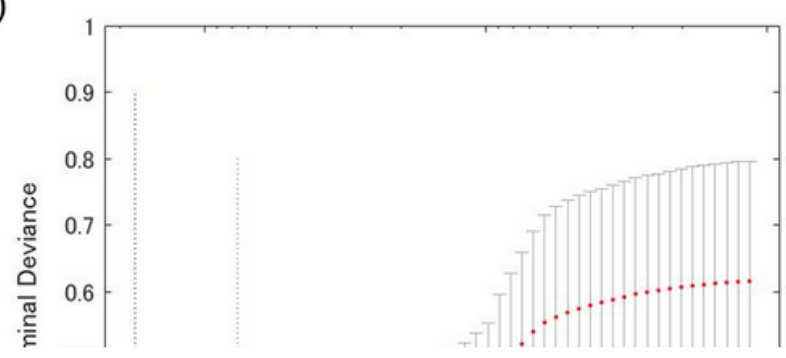

b)

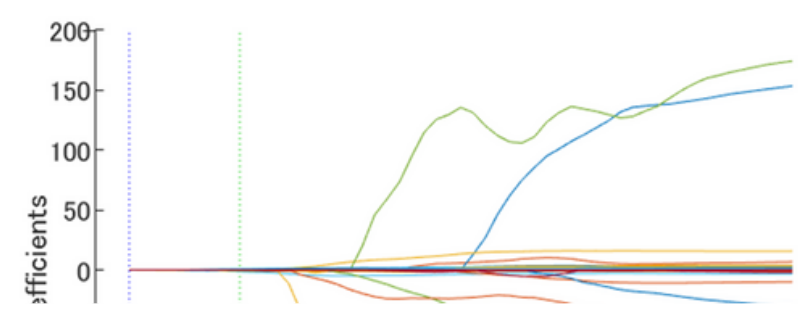

Figure 4

Radiomics features were selected using the LASSO regression. a) Tuning penalization parameter $(\lambda)$ and minimum criterion in the LASSO model. The binomial deviance was plotted against $\log (\lambda)$. b) LASSO coefficient profiles of the 4185 radiomics features. The green line showed the optimal lambda in the LASSO analysis with the least partial likelihood deviance. 


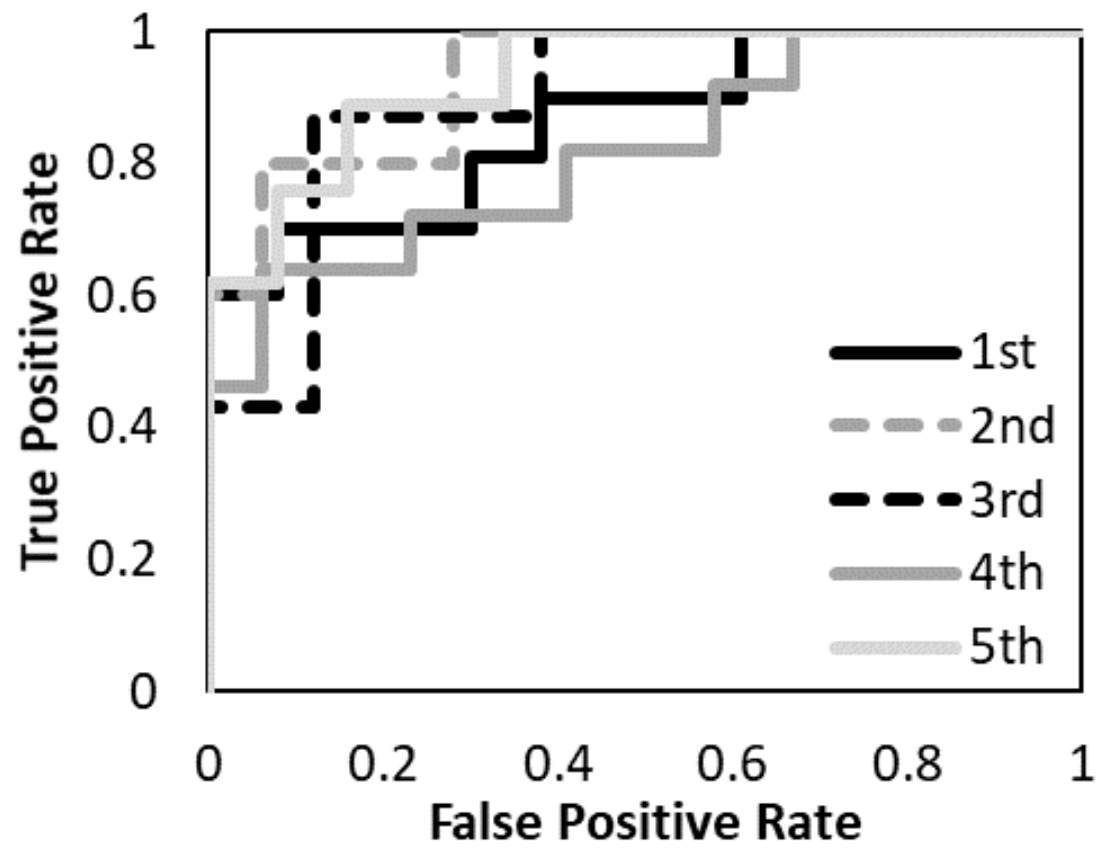

Figure 5

The performance of the predictive model with the T1-weighted MRI image was evaluated according to the ROC metrics.

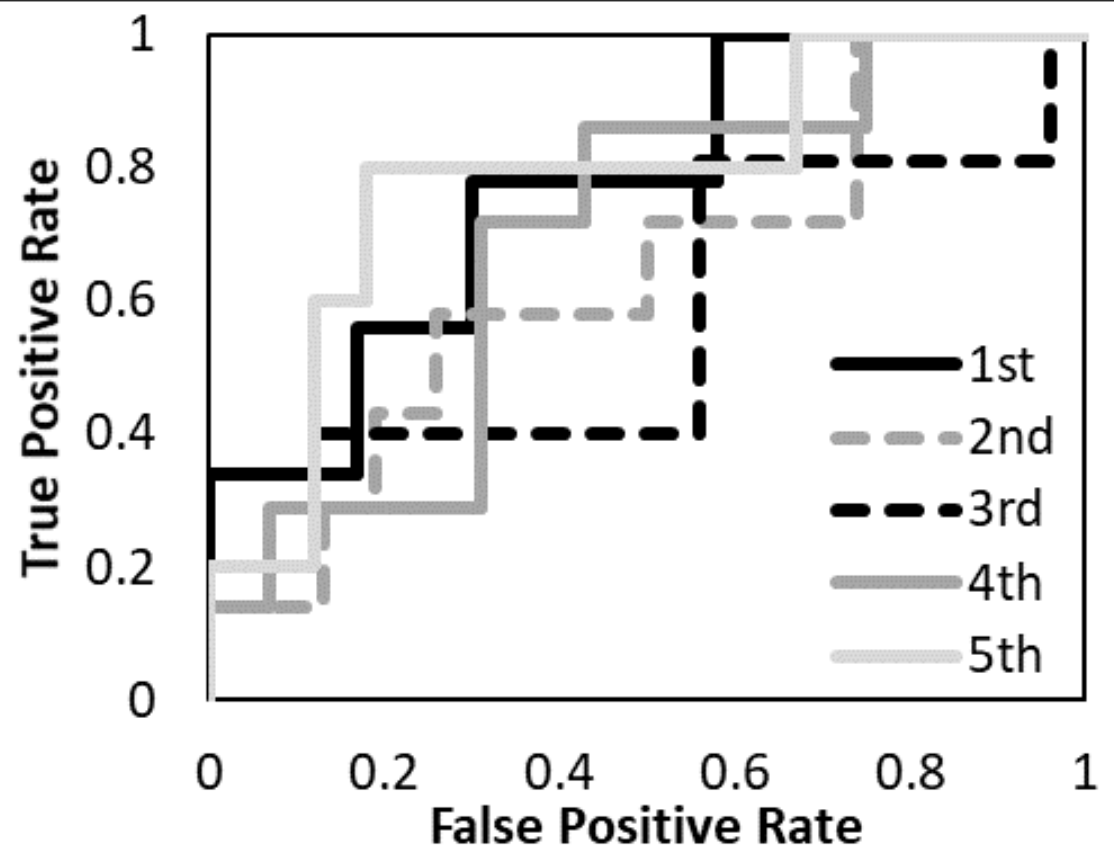

Figure 6

The performance of the predictive model with the T2-weighted MRI image was evaluated according to the ROC metrics. 


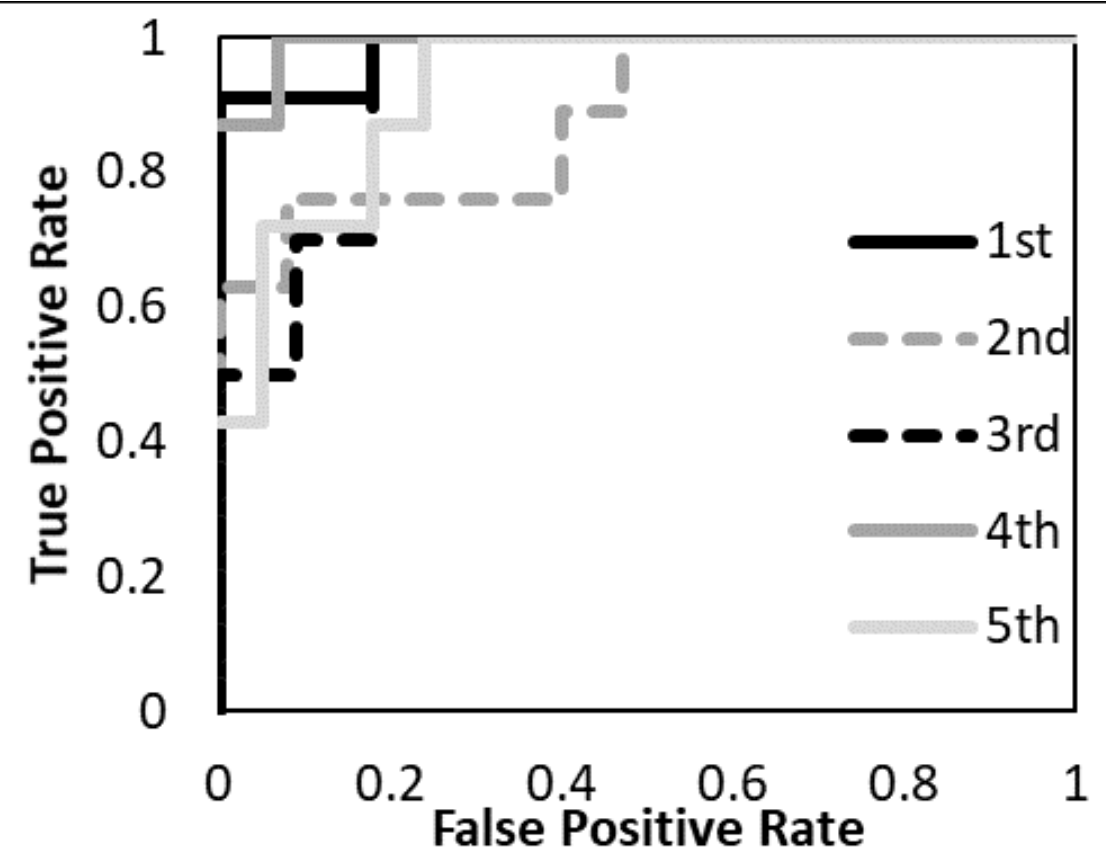

Figure 7

The performance of the predictive model with T1- and T2- weighted MRI images was evaluated according to the ROC metrics.

\section{Supplementary Files}

This is a list of supplementary files associated with this preprint. Click to download.

- SuplementalTable20211105.docx 